\title{
Advanced Wireless Communications and Mobile Computing Technologies for the Internet of Things
}

\author{
Haiyu Huang $\mathbb{D},{ }^{1,2}$ Kejie Lu, ${ }^{3}$ Giovanni Pau $\mathbb{D},{ }^{4}$ Yong Ren, ${ }^{5}$ and Pai-Yen Chen ${ }^{6}$ \\ ${ }^{1}$ Department of Electrical and Computer Engineering, University of Texas, Austin, TX, USA \\ ${ }^{2}$ Maxim Integrated Inc., San Jose, CA, USA \\ ${ }^{3}$ Department of Electrical and Computer Engineering, University of Puerto Rico, Mayagüez, PR, USA \\ ${ }^{4}$ Faculty of Engineering and Architecture, Kore University of Enna, Enna, Italy \\ ${ }^{5}$ Department of Electronic Engineering, Tsinghua University, Beijing, China \\ ${ }^{6}$ Department of Electrical and Computer Engineering, Wayne State University, Detroit, MI, USA \\ Correspondence should be addressed to Haiyu Huang; harryhuang@utexas.edu
}

Received 18 March 2018; Accepted 21 March 2018; Published 24 April 2018

Copyright (c) 2018 Haiyu Huang et al. This is an open access article distributed under the Creative Commons Attribution License, which permits unrestricted use, distribution, and reproduction in any medium, provided the original work is properly cited.

Since its origination from P\&G and MIT Auto-ID Center in 1999, the term "Internet of Things" (IoT) has been elevated from a specific application concept, based on RFID, to a vastly prominent phrase that represents the general direction to the future of many important aspects of human life. From the application perspective, all the major industries and sectors are now experiencing a certain level of paradigm shift thanks to IoT. For instance, the healthcare system is moving from hospital-centered care to distributed omnipresent care; the transportation is gradually replacing human involvement with driverless technologies and vehicle-to-vehicle (V2V) communications; in manufactory, Industry 4.0, which is empowered by IoT, is taking the leading role in changing the face of factories everywhere in the world. From technology perspective, trending research/development fields in electrical and computer engineering, such as Device-to-Device Network, Wireless Sensor Networks, 5G, LoRaWAN, Bluetooth LE, MIMO, deep learning, and distributed low power computing, have all been directly or indirectly contributing to the evolution of IoT.

Due to the timeliness and importance of the topics, we received a large number of submissions. In the review process, each paper was reviewed by multiple experts in relevant fields. After a rigorous two-round review process, we decided to accept 10 excellent articles addressing cutting-edge wireless communications and mobile computing technologies and applications around the latest trend of IoT. Since the topics of articles cover broad technology scopes, we will introduce them below according to the themes of IoT applications.

The first theme is device-to-device (D2D) communication. In this special issue, we have two papers investigating different D2D aspects. Specifically, in "Clustering Optimization for Out-of-Band D2D Communications," the authors focused on clustering optimization algorithms for Out-ofBand D2D communication. The results showed that wellknown clustering algorithms can be employed to determine the cluster head which provides a near-optimal solution for throughput in channels between the cluster head and its members.

In "Performance Analysis of Three-Dimensional Clustered Device-to-Device Networks for Internet of Things," $\mathrm{H}$. Jung and I.-H. Lee aimed at modeling and analyzing clustered D2D networks in three-dimensional space for scenarios where devices are stacked vertically and dispersed in the horizontal plane.

The second theme is low power/short distance wireless communications for IoT. In this issue, we accepted four papers that are related to low power and/or short distance wireless communication technologies. In "Maximum Power Plus RSSI Based Routing Protocol for Bluetooth Low Energy Ad Hoc Networks," the authors proposed an energyconserving multihop routing protocol for Bluetooth Low Energy. 
Next, in "A High Throughput Anticollision Protocol to Decrease the Energy Consumption in a Passive RFID System," the aim is an anticollision protocol that can solve tag collision problem in UHF RFID.

The paper "An Adaptive Scheduler for Real-Time Operating Systems to Extend WSN Nodes Lifetime" introduced an adaptive scheduler for RTOS used in WSN sensor node to reduce energy consumption and thus increase the lifetime of battery powered WSN sensor node.

In the paper "A Dual Key-Based Activation Scheme for Secure LoRaWAN," the authors focused on the security issue of LoRaWAN, which is one of the most promising Low Power Wide Area Network technologies for IoT, and they proposed a dual key-based activation scheme for LoRaWAN to improve its security.

The third theme in our special issue is MIMO and beamforming for $5 \mathrm{G}$ and IoT. There are three relevant papers in this category. The paper "5G MIMO Conformal Microstrip Antenna Design” demonstrated eight-element microstrip MIMO conformal antennas at $35 \mathrm{GHz}$ well suited for the $5 \mathrm{G}$ MIMO communication.

In "Pipeline Implementation of Polyphase PSO for Adaptive Beamforming Algorithm," the authors investigated a low hardware cost realization of a partial Particle Swarm Optimization algorithm for an adaptive beamformer.

The paper "Optimized Power Allocation and Relay Location Selection in Cooperative Relay Networks" focused on power allocation optimization in cooperation communication used in MIMO.

Finally, yet importantly, the last theme is cyberphysical systems for IoT. Here we have one paper "SVM-Based Dynamic Reconfiguration CPS for Manufacturing System in Industry 4.0," in which a cyberphysical system framework was designed using learning algorithm SVM to support Industry 4.0 .

Haiyu Huang

Kejie Lu

Giovanni Pau

Yong Ren

Pai-Yen Chen 


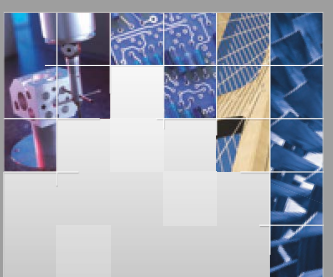

\section{Enfincering}
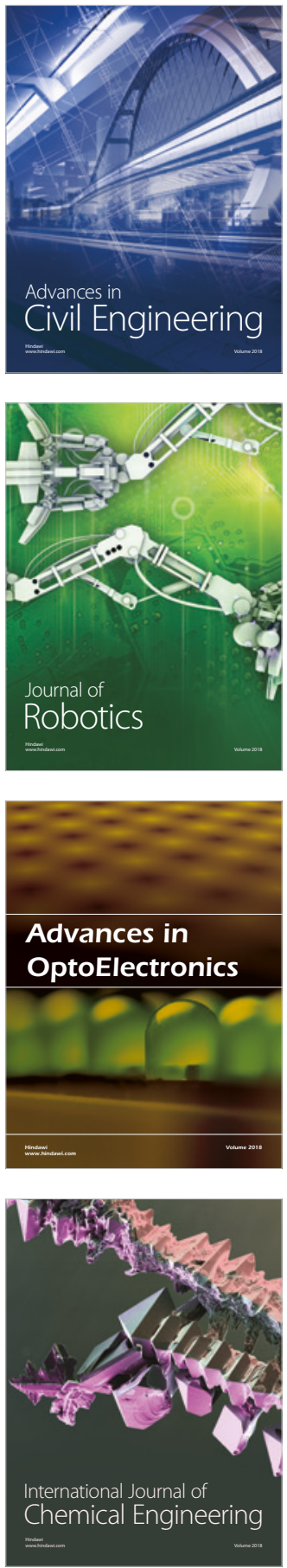

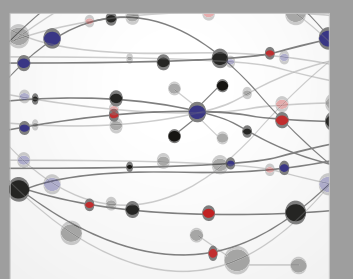

\section{Rotating \\ Machinery}

The Scientific World Journal

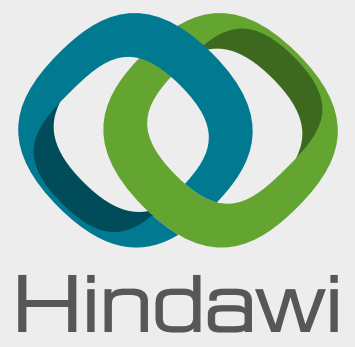

Submit your manuscripts at

www.hindawi.com
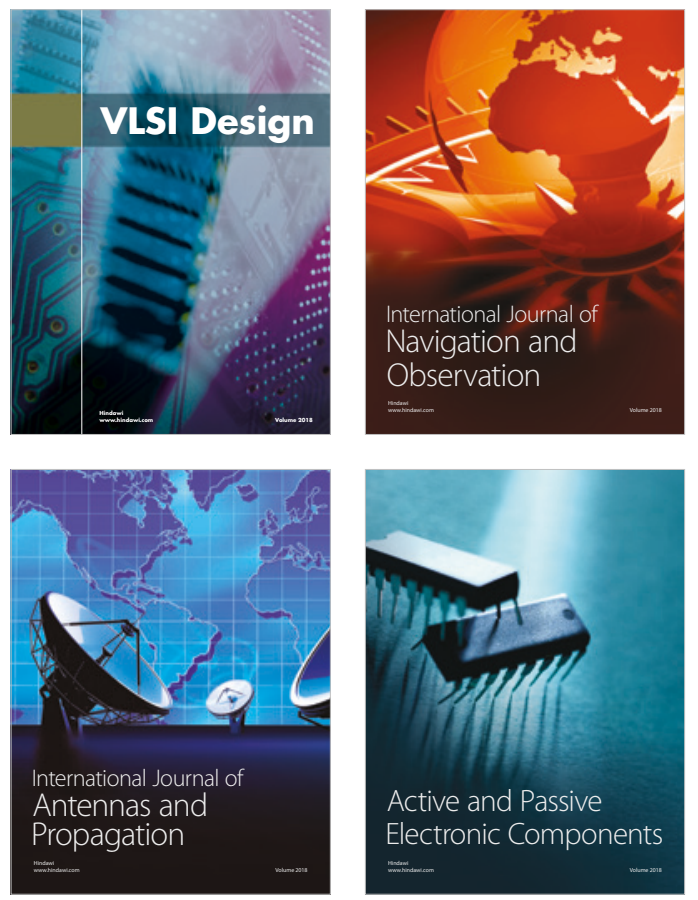
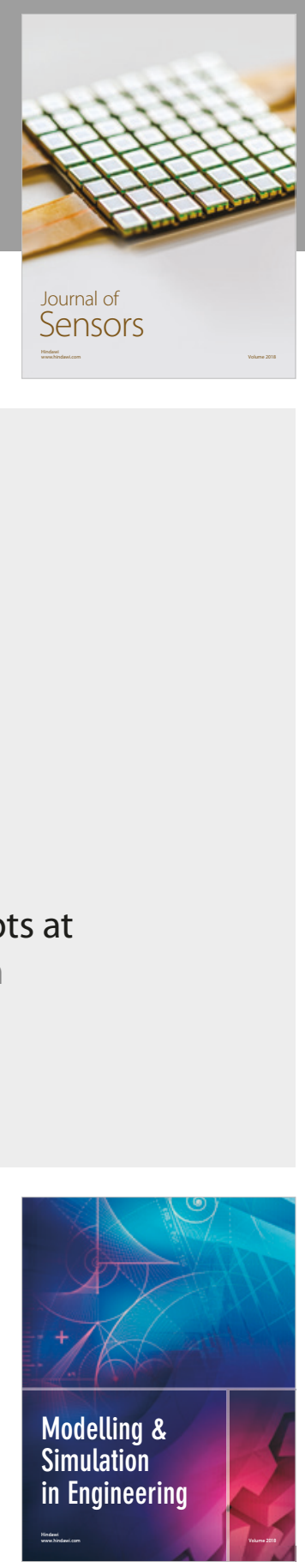

\section{Advances \\ Multimedia}
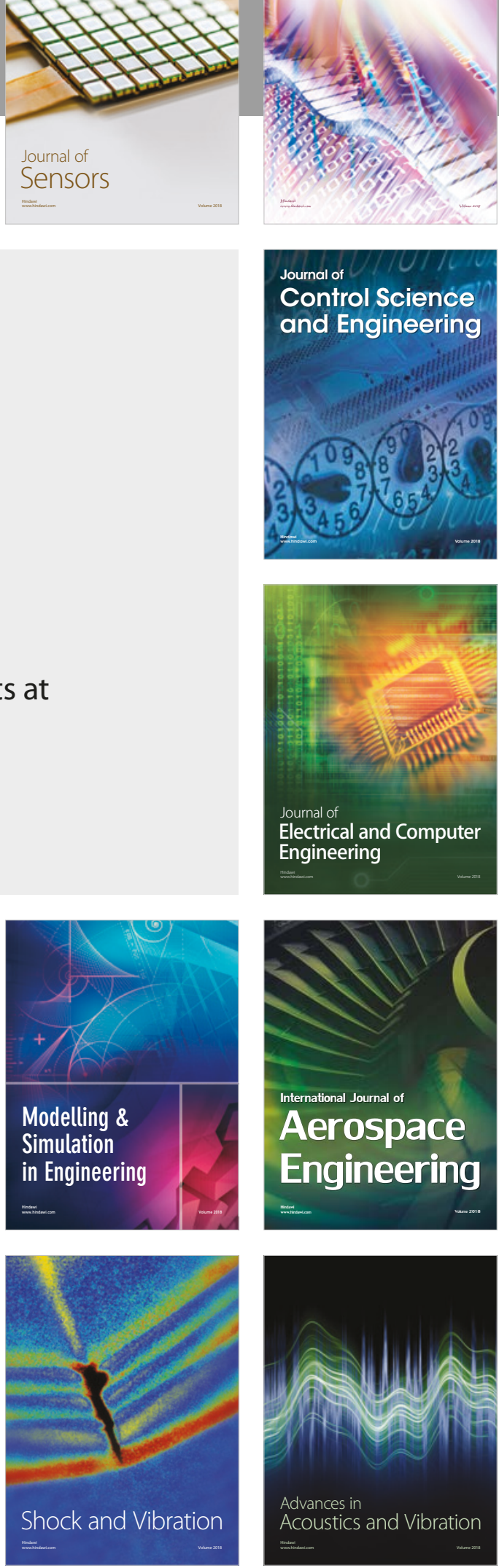